\title{
Calculation of the spectra of characteristic electron losses in indium bromide
}

\author{
M.O. Kolinko ${ }^{1}$, O.V. Bovgyra ${ }^{2}$ \\ ${ }^{I}$ Lviv State Agrarian University, 79240 Lviv-Dublyany, Ukraine \\ ${ }^{2}$ Ivan Franko Lviv National University, \\ 1, Universytetska str., 79602 Lviv, Ukraine
}

\begin{abstract}
For the first time, the fundamental optical functions of single crystals of layered indium bromide are calculated on the basis of polarization-dependent reflection spectra at the liquid helium temperature in the energy region of $2-30 \mathrm{eV}$. Features of the spectra of bulk and surface characteristic losses are examined, and the bulk and surface plasmon energies have been obtained. The identification of the genetic origin of the principal structures in the spectra is accomplished. The correlation between the energies of long-wave plasmons and the energies of reflectivity minimum has been determined.
\end{abstract}

Keywords: surface and bulk plasmons, reflection.

Manuscript received 07.02.07; accepted for publication 27.09.07; published online 31.10.07.

\section{Introduction}

In experimental studies of the electron structure of crystals, the investigation of reflection, transmission, and photoemission spectra is widely used. The use of the light sources enables determining the contribution of the transverse components of transitions, whereas the beams of fast charged particles are usually used to obtain the longitudinal components. From the energy loss function $W$, which is measured experimentally, the functions of bulk characteristic losses $-\operatorname{Im}\left(\varepsilon^{-1}\right)$ and surface characteristic losses $-\operatorname{Im}(1+\varepsilon)^{-1}$ are derived using a special technique. Usually the loss energy function $W$ has rather complicated structure that is formed by the contributions of interband transitions, as well as plasmon excitations and exciton effects, and the form of the spectrum depends on both the orientation of a sample and the electron beam intensity. Whereas the spectral resolution is not better than $0.5 \mathrm{eV}$, and the process of derivation of the loss functions $-\operatorname{Im}\left(\varepsilon^{-1}\right)$ and $-\operatorname{Im}(1+\varepsilon)^{-1}$ is a complicated ambiguous procedure which is accompanied by a number of approximations and simplifications, determining the energy and obtaining the true plasmon spectra are problematic even for well-investigated crystals.

Due to difficulties of the direct measurement and the processing of the spectra of characteristic losses, the special actuality gains the technique of its determination on the basis of experimental optical reflection spectra measured in a wide energy region. To derive the loss functions $-\operatorname{Im}\left(\varepsilon^{-1}\right)$ and $-\operatorname{Im}(1+\varepsilon)^{-1}$, the integral Kramers-Kronig [1-5] relations are used.
The aim of this paper is to determine the spectra of characteristic losses by means of calculations and experimental studies and to obtain the energies of surface and bulk plasmons in indium bromide single crystals.

Indium mono-bromide belongs to the family of halides of metals of the third group, which have been actively investigated during last two decades because of their applications in laser and infrared engineering as well as in fiber optics. Recently, they have found industrial application as materials for producing the photodetectors used in the scintillation spectroscopy of nuclear radiation as well [6]. However, the properties of layered halides of metals of the third group can hardly be explained without information about their energy structure and the contribution of ion terms into the formation of optical spectra.

Since the energy band structure of indium bromide has not been determined yet, obtaining the spectra of optical functions in the core region of fundamental absorption, which show the structure of the valence band and the conduction band, is the most efficient way of revealing the physical mechanisms of processes running in these compounds.

\section{Procedure of measurements}

As a source of synchrotron radiation, the accelerator with an energy of $600 \mathrm{MeV}$ has been used. The operating pressure was about $1.3 \cdot 10^{-6} \mathrm{~Pa}$. The incident and reflected radiations were analyzed with sodium salicy- 
late. As a monochromator, we used a Seya Namioka vacuum spectrometer. Measurements were carried out for three incidence angles $\left(8,12\right.$, and $\left.16^{\circ}\right)$, and the data obtained were analyzed using a specially constructed computer code. Spectral resolution was $0.7 \mathrm{~nm} / \mathrm{mm}$. The absolute values of reflectivity have been obtained using a germanium single crystal. Measurements were carried through in two temperature regimes: at room temperature and the liquid helium temperature.

Parent material was synthesized in vacuum in the reaction of high-quality pure metallic indium with liquid bromine of guaranteed quality in a soldered ampoule. Synthesis was carried out under an optimum temperature of $250{ }^{\circ} \mathrm{C}$ with surplus of metal in order to prevent the formation of three-bromide. Obtained $\mathrm{InBr}$ after distillation was subjected to the zone refining during several dozens of hours. Single crystals that were grown up with the use of the Bridgman method were split only in one definite direction, the surfaces that were formed having the typical mirror-like luster and demanding no further processing.

$\mathrm{X}$-ray analysis has confirmed that indium bromide crystallizes [7] as a layered structure of the TII-type with space group $\mathrm{Cmcm}$ and has revealed that single crystals split up normally to the $b$-axis, hence the surfaces contain two crystallographic directions $a$ and $c$. Orientation of the samples in relation to the crystallographic axes was carried out by the use of cleavage planes and was verified by means of an X-ray diffractometer DRON-5.

\section{Calculation technique}

The series of fundamental optical constants (functions) calculated on the basis of experimental reflection spectra $R(E)$ includes: the real $\varepsilon_{1}$ and imaginary $\varepsilon_{2}$ parts of the dielectric function; the effective number of valence electrons $N_{\text {eff }}(E)$ that are actual up to a certain energy value; the effective dielectric function $\varepsilon_{\text {eff }}$; characteristic losses of bulk $-\operatorname{Im}\left(\varepsilon^{-1}\right)$ and surface $-\operatorname{Im}(1+\varepsilon)^{-1}$ plasmons; absorptivity $\kappa$ and refraction coefficient $n$; function $\varepsilon_{2} E^{2}$ that is proportional to the reduced density of states provided that the strength of oscillators is equal to unity.

The functions of plasmons can be determined either from the dielectric functions $\varepsilon_{1}$ and $\varepsilon_{2}$, or by using the refraction coefficient $n$ and absorptivity $\kappa$ :

$-\operatorname{Im}\left(\varepsilon^{-1}\right)=\varepsilon_{2}\left(\varepsilon_{1}^{2}+\varepsilon_{2}^{2}\right)^{-1}=2 n \kappa\left(n^{2}+\kappa^{2}\right)^{-2}$,

$-\operatorname{Im}(1+\varepsilon)^{-1}=\varepsilon_{2}\left[\left(\varepsilon_{1}+1\right)^{2}+\varepsilon_{2}^{2}\right]^{-1}=$

$=2 n \kappa\left[\left(n^{2}+\kappa^{2}\right)^{2}+2\left(n^{2}-\kappa^{2}\right)+1\right]^{-1}$.

In order to obtain the optical functions, we used the technique that had been approved not once. While computing the phase integral, the extrapolation into the energy region that lies beyond measurements was carried out by power-law functions, with parameters found by solving the determinant boundary conditions in the transparency region where the phase is known to be equal to zero, $\Theta(E) \equiv 0$.

\section{Optical spectra and energies of plasmons}

The initial data for calculations of the characteristic loss function were the experimental measurements of lowtemperature polarization-dependent reflection spectra that are shown in Fig. 1. The plastic properties of this compound caused the difficulties in obtaining the sample surfaces of high quality to be used in investigations of $\mathbf{E} \| \mathbf{b}$ polarization.

The spectra for both polarizations exhibit a complicated structure which is formed by the significant number of peaks, especially in the region up to $15 \mathrm{eV}$, whereas the reflectivity drops sharply at higher energies. The behavior of the curve $R(E)$ in this region is violated by two minima about 4.6 and $7.5 \mathrm{eV}$.

The exciton structure of spectra reminds that of alkali-halide crystals. However, $\mathrm{InBr}$ is distinguished by having a large dielectric constant, the edge of fundamental absorption being in the shorter wave region and the parameters of unit cell being less, hence the exciton features of the spectra are still less distinct.

In general, the spectra in Fig. 1 can be partitioned into 4 regions that differ in the character and behavior of the reflectivity curve: up to $\approx 4.7 \mathrm{eV}$, from 4.7 to $7.5 \mathrm{eV}$, from 7.5 to $16 \mathrm{eV}$, and higher than $16 \mathrm{eV}$. Let's note that, in all the energy regions, the reflectivity for $\mathbf{E} \| \mathbf{c}$ polarization is larger than that for $\mathbf{E} \| \mathbf{a}$ polarization except for two narrow intervals $4.7-6 \mathrm{eV}$ and $14-15 \mathrm{eV}$. If the layered compounds show the dichroism of optical properties between the directions which lie in the wafer plane and that direction which is perpendicular to this plane, then, in the case of indium bromide, the anisotropic nature of a crystal exhibits itself in the plane of the layer as well, as can be seen from Fig. 1.

When increase in the temperature up to room one, the abrupt change of the $R(E)$ spectrum form is observed, which is accompanied by the temperaturecaused break-down of excitons. This fact is an evidence of the effect of the phonon subsystem on the interband electron transitions, whereas the existence of excitons with high energies is a criterion of strong electronphonon correlation.

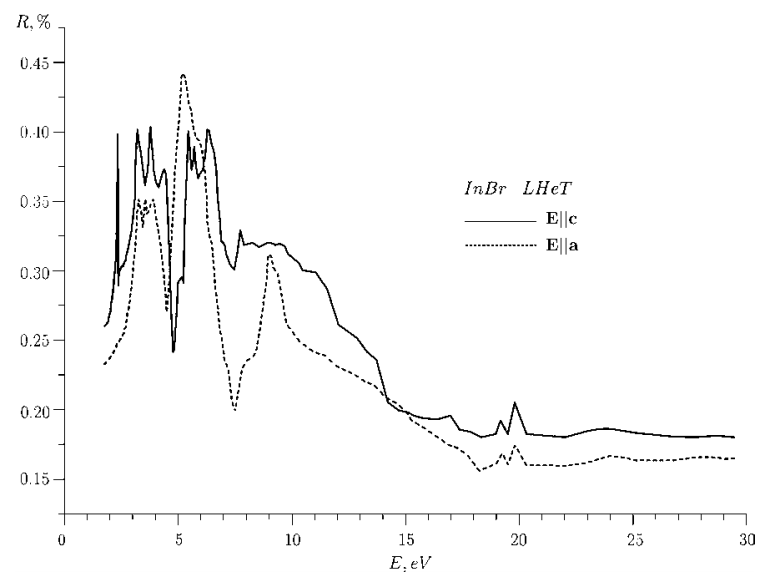

Fig. 1. Polarization-dependent reflection spectra of indium bromide single crystals. 
In the calculated dependences of the dielectric function, the change of the sign of $\varepsilon_{1}$ is observed in the region of $6.3-6.7 \mathrm{eV}$, that is the evidence of the probable appearance of collective phenomena. The excitation of plasmon oscillations in this region is corroborated by the maxima in the spectra of the characteristic energy loss function $-\operatorname{Im}\left(\varepsilon^{-1}\right)$ near $7 \mathrm{eV}$ in Fig. 2.

The masking effect on the weak exhibition of the structure of fundamental plasmons, that can be observed in the region 13-14 eV, where there are no structures in the $R(E)$ spectra, is exerted by interband transitions of valent $4 s$ halogen electrons and core $4 \mathrm{~d}$ metal electrons. Their levels, according to the photoelectron spectroscopy data [8], are localized near -14 and $-17 \mathrm{eV}$ (the zero energy level being fixed at the valence band top) respectively, and they merge together into a wide band at $\approx 8 \mathrm{eV}$, in contrast to indium iodide single crystals where the corresponding maxima are energetically localized [9]. The exciton $4 d$ In effect that is observed in Fig. 1 in the region near $19 \mathrm{eV}$ causes the further broadening of the plasmon peak. The theoretical estimation of the energy of fundamental plasmons gives a value of $14.5 \mathrm{eV}$ that is in good agreement with experimental data.

The existence of plasmons of two types is due to the division of valence electrons into two groups that are formed by $\pi$ - and $\sigma$-electrons in particular, with the degree of such a division being determined by the anisotropy of the crystal lattice. As is well known [9-11], the $s$-electrons of halogen completely fill two lowest valence bands in the halides of the metals of the third group. The remaining bands of the valence complex, which are separated from the above-mentioned ones by the energy interval of about 7-8 eV, are formed mostly by $p$-electrons of the metal and the halogen. Two types of energetically separated plasmons had already been observed for the layered compounds in the spectra of optical functions of graphite [12] and molybdenum dichalcogenides [13].

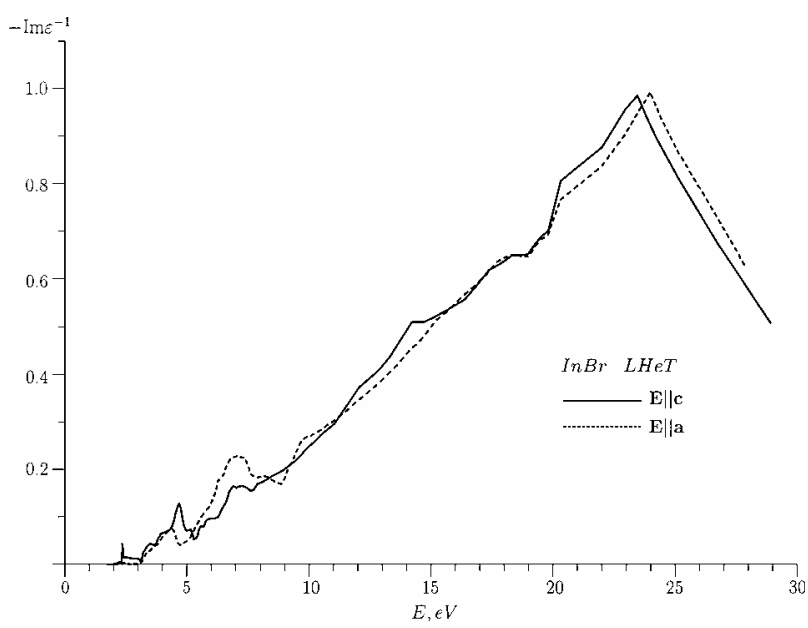

Fig. 2. Electron energy loss function $-\operatorname{Im}\left(\varepsilon^{-1}\right)$ of $\operatorname{InBr}$ derived from reflection spectra through the Kramers-Kronig analysis.

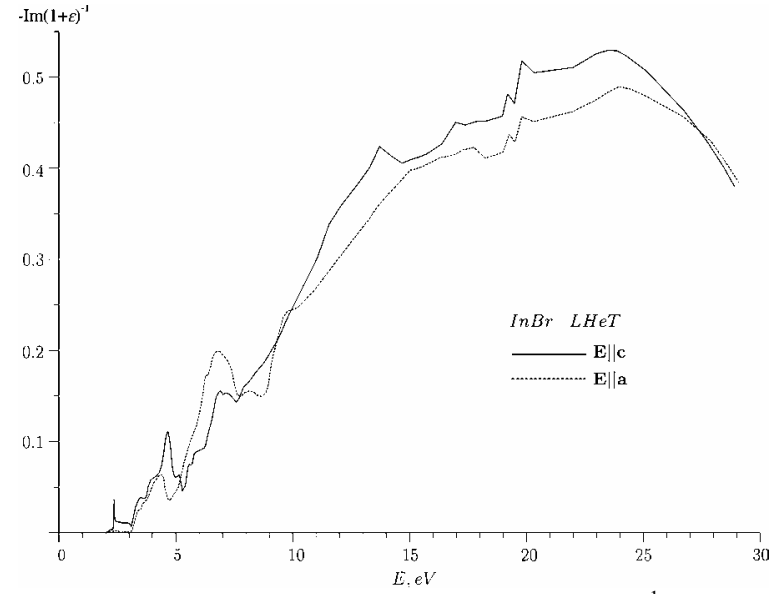

Fig. 3. Electron energy loss function $-\operatorname{Im}(1+\varepsilon)^{-1}$ of indium bromide derived from reflection spectra through the KramersKronig analysis.

Direct measurements of the characteristic energy losses using light transmission would have advantages in this case and would have allowed us, on the one hand, to abstract from the conditions of the surface of this, generally speaking, hygroscopic compound and, on the other hand, to measure more exactly the excitations with energies higher than $15 \mathrm{eV}$.

It should be noted that, for the solids with large dielectric constants, there often can be observed that the peaks of characteristic energy $\operatorname{losses}-\operatorname{Im}\left(\varepsilon^{-1}\right)$ lead the peaks of $\varepsilon_{1}, \varepsilon_{2}$ (e.g., in isoelectronic and isostructural thallium iodide [14]), in contrast to the our ordinary situation when the maxima of $\varepsilon_{1}, \varepsilon_{2},-\operatorname{Im}\left(\varepsilon^{-1}\right)$ follow each other in all the energy region with increase in the energy as those in alkali-halide compounds. The shift between the maxima of characteristic energy losses and the maxima of the imaginary part of the dielectric function $\varepsilon_{2}$, that governs the magnitude of the longitudinaltransverse split of the transitions, is equal to $0.1-0.4 \mathrm{eV}$.

The spectra of the surface characteristic losses for indium bromide single crystals are shown in Fig. 3. As was predicted by theory, the magnitude of the surface characteristic losses as well as the energy of surface plasmons are significantly less than the corresponding values for bulk plasmons, especially in the high-energy region.

The shift of surface plasmons of the first type to the long-wave side of the spectrum is equal to $0.2 \mathrm{eV}$ in comparison with bulk plasmons. For plasmons of the main type, this shift appears to be equal to almost $1 \mathrm{eV}$. In general, the method of characteristic losses, based on the calculations from the reflection spectra data, proved to be a powerful means of revealing the division of valence electrons into two groups and the appearance of two types of plasmons.

\section{Summary}

In this paper, the fundamental optical functions of layered indium bromide single crystals have been calcu- 
lated for the first time on the basis of the polarizationdependent reflection spectra measured at the liquid helium temperature in the energy region of $2-30 \mathrm{eV}$. The exact spectra of bulk and surface charac-teristic electron losses have been registered, and the energies of plasmons have been determined.

For the first time, two types of bulk and surface plasmons are revealed in the layered crystals of indium bromide, which points out at the distinct division of valence electrons into two groups.

The identification of the genetic origin of the principal structures of the spectra was carried out, and the correlation between the energies of long-wave plasmons and the minimum reflectivity energies has been obtained.

\section{References}

1. R. Klucker and U. Nielsen, Kramers-Kronig analysis of reflection data // Computer Phys. Comm. 6, p. 187-192 (1973).

2. D.M. Roessler, Kramers-Kronig analysis of reflection data // Brit. J. Appl. Phys. 16, No. 8, p. 11191123 (1965).

3. K. Jezierski, A linear-equation algorithm for reflectivity extrapolation determination in Kramers-Kronig analysis // J. Phys. C: Solid State Phys. 17, No. 3, p. 475-484 (1984).

4. K. Jezierski, Improvement in Kramers-Kronig analysis of reflection spectra // J. Phys. C: Solid State Phys. 19, No. 8, p. 2103-2112 (1986).

5. B. Velicky, The use of the Kramers-Kronig relations in determining optical constant // Czech. J. Phys. 11B, p. 787-797 (1961).
6. K.S. Shah, L.P. Moy, J. Zhang, S. Medrick, F. Olschner, M.R. Squillante, InI photodetectors for scintillation spectroscopy // SPIE Proc. 1734, p. 161-165 (1992).

7. T. Staffel and G. Meyer, The mono-, sesqui-, and dibromides of indium: $\mathrm{InBr}, \mathrm{In}_{2} \mathrm{Br}_{3}$, and $\mathrm{InBr}_{2} / / Z$. Anorg. Allg. Chem. 552, p. 113-122 (1987).

8. L. Porte, Indium (I) halides valence band structure and stability in the $\mathrm{A}^{3} \mathrm{~B}^{7}$ compounds: an XPS study // Solid State Communs 42, No. 2, p. 85-88 (1982).

9. M.I. Kolinko, Density of states of InI: theoretical and experimental investigation // Phys. Rev. B 55, No. 7, p. 4007-4010 (1997).

10. M.I. Kolinko, I.V. Kityk, A.S. Krochuk, Band energy parameters and density functions of the orthorhombic TlI // J. Phys. Chem. Solids 53, No. 10, p. 1315-1320 (1992).

11. M.I. Kolinko, Electronic energy-structure calculations of orthorhombic InI single crystals // J. Phys. Condens. Matter. 6, No. 1, p. 183-202 (1994).

12. E.A. Taft and H.R. Philipp, Optical properties of graphite // Phys. Rev. 138, No. 1A, p. 197-202 (1965).

13. A.N. Timoshkin, V.Val. Sobolev and V.V. Sobolev, Electron energy loss spectra of molybdenum dichalcogenides // Fiz. Tverd. Tela 42, No. 1, p. 3739 (2000) (in Russian).

14. J. Frandon and B. Lahaye, Pertes caracterisatiques des electrons dans $\mathrm{TlCl}, \mathrm{TlBr}$, TlI et calcul des fonctions optiques entre 3 et $25 \mathrm{eV} / / J$. de Physique 33, No. 2-3, p. 229-235 (1972). 